\title{
Evolution of a local Boundary Detector for Natural Images via Genetic Programming and Texture Cues
}

\author{
Ilan Kadar \\ Dept. of Computer Science \\ Ben-Gurion University \\ Beer-Sheva, Israel \\ ilankad@cs.bgu.ac.il
}

\author{
Ohad Ben-Shahar \\ Dept. of Computer Science \\ Ben-Gurion University \\ Beer-Sheva, Israel \\ ben-shahar@cs.bgu.ac.il
}

\author{
Moshe Sipper \\ Dept. of Computer Science \\ Ben-Gurion University \\ Beer-Sheva, Israel \\ sipper@cs.bgu.ac.il
}

\begin{abstract}
Boundary detection constitutes a crucial step in many computer vision tasks. We present a learning approach for automatically constructing high-performance local boundary detectors for natural images via genetic programming (GP). Our GP system is unique in that it combines filter kernels that were inspired by models of processing in the early stages of the primate visual system, but makes no assumptions about what constitutes a boundary, thus avoiding the need to make ad hoc intuitive definitions. By testing our evolved boundary detectors on a highly challenging benchmark set of natural images with associated human-marked boundaries, we show performance that outperforms most existing approaches.
\end{abstract}

\section{Categories and Subject Descriptors}

I.4.6 [Computing Methodologies]: Image Processing and Computer Vision-Segmentation

\section{General Terms}

Algorithms, Measurement, Performance, Design

\section{Keywords}

Boundary Detection, Computer Vision, Machine Learning, Evolutionary Algorithms

\section{INTRODUCTION}

Boundary detection in images is a fundamental problem in computer vision. The performance of many high-level computer vision tasks, such as segmentation and object recognition, is highly dependent upon the boundary map of an image. A boundary is a contour in the image plane that represents a change in the pixel's "ownership" from one object or surface to another. In general, there are different types of boundaries: for example, those formed between two regions with an abrupt change in the image brightness, and those formed between two regions with a change in the texture. Clearly, boundaries in natural images are marked by change both in brightness and texture. There are

Copyright is held by the author/owner(s). GECCO'09, July 8-12, 2009, Montréal, Québec, Canada. ACM 978-1-60558-325-9/09/07. some attempts in computer vision to address both brightness and texture cues using complex and computationally intensive schemes [5]. In contrast, humans have an outstanding ability to detect boundaries pre-attentively, and hence very fast. Correspondingly, evidence from behavioral science and neuroscience strongly suggests that this process occurs in early stages of visual processing. This paper presents an approach that aims to use genetic programming (GP) as a learning framework for evolving detectors. The detectors are evaluated against human-marked boundary maps in order to accurately detect and localize boundaries in grayscale natural images. The evolving programs use both linear and non-linear operators to combine multiple cues from the early stages in the visual cortex. The presented learning framework was developed based on insights from our recent work [3] with the critical improvement of incorporating texture cues. Our results show that this approach is highly effective at automatically generating boundary detectors. By testing the evolutionary algorithm on a highly challenging benchmark set of natural images with associated human-marked boundaries, we show performance to be quantitatively human competitive [4].

\section{METHOD}

We present a visual learning approach to automatically construct a boundary detector using $G P$ and texture cues. Each individual in the GP population represents a candidate boundary cue, which is then combined with a texture gradient cue into a single detector on a trained logistic regression classifier. Fitness is defined as the F-measure, computed for a set $T=\left\{I_{i}\right\}$ of $n$ images taken from the training set of the Berkeley data set. The terminal set is image independent, such that the terminals for image $I_{i}$, given as an array of matrices, are the convolution of the image $I_{i}$ with filter kernels tuned to various orientations. These filter kernels are inspired by models of processing in the early stages of the primate visual system, which model both odd- and evensymmetric simple cell receptive fields at various orientations (see Figure 1). The function set contains both unary and binary functions. The input and output of all functions are arrays of length $N$ of data matrices with the same size as images in $T$ (see Table 1). In order to measure the degree to which texture varies at a location $(x, y)$ in direction $\theta$, we used a texture gradient approach inspired by Martin and Malik [5]. 


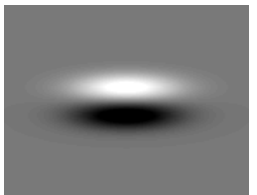

(a)

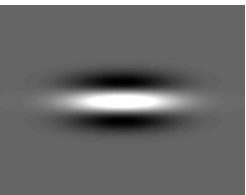

(b)
Figure 1: (a) Odd-symmetric simple cell with preferred orientation $0^{\circ}$. (b) Even-symmetric simple cell with preferred orientation $0^{\circ}$.

Table 1: Function set

\begin{tabular}{ll}
\hline \hline$M_{\forall i \in 1 \ldots N}=\operatorname{Add}\left(M_{1_{i}}, M_{2_{i}}\right)$ & Matrix addition \\
\hline$M_{\forall i \in 1 \ldots N}=\operatorname{Sub}\left(M_{1_{i}}, M_{2_{i}}\right)$ & Matrix subtraction \\
\hline$M_{\forall i \in 1 \ldots N}=\operatorname{Mul}\left(M_{1_{i}}, M_{2_{i}}\right)$ & Matrix multiplication \\
\hline$M_{\forall i \in 1 \ldots N}=\operatorname{Max}\left(M_{1_{i}}, M_{2_{i}}\right)$ & $\begin{array}{l}\text { Largest elements taken } \\
\text { from } M_{1_{i}} \text { or } M_{2_{i}}\end{array}$ \\
\hline$M_{\forall i \in 1 \ldots N}=\operatorname{Min}\left(M_{1_{i}}, M_{2_{i}}\right)$ & $\begin{array}{l}\text { Smallest elements taken } \\
\text { from } M_{1_{i}} \text { or } M_{2_{i}}\end{array}$ \\
\hline$M_{\forall i \in 1 \ldots N}=\operatorname{Pow2}\left(M_{1_{i}}\right)$ & Elements are 2 raised to the \\
& power $M_{1_{i}}$ \\
\hline$M_{\forall i \in 1 \ldots N}=\operatorname{Sqrt}\left(M_{1_{i}}\right)$ & Square root of each element \\
& of $M_{1_{i}}$ \\
\hline$M_{\forall i \in 1 \ldots N}=M_{(i+N / 2) \% N}$ & R90 $\left(90^{\circ}\right.$ Rotation) \\
\hline$M_{\forall i \in 1 \ldots N}=M_{1_{i}} * K_{o d d_{i}}$ & $M_{1_{i}}$ convolved with ori- \\
& ented odd symmetric kernel \\
\hline \hline
\end{tabular}

\section{RESULTS}

The output of each detector for a given image $I_{i}$ is a soft boundary map, which provides the probability of a boundary at each image location. The fittest individual generated with our approach is presented in Figure 2. The presented

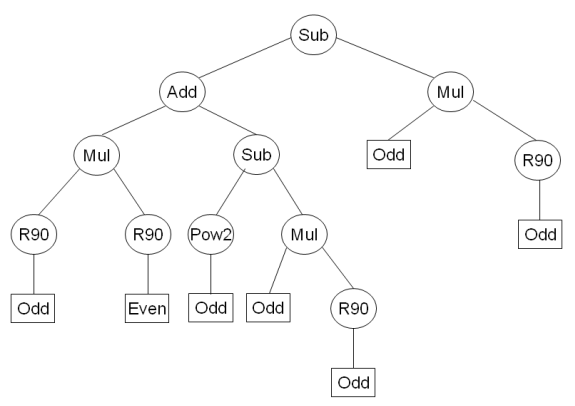

Figure 2: The fittest individual generated with our approach, with performance $\boldsymbol{F}$-measure $=0.62$.

detector was tested on the Berkeley test set of 100 images [6], and the overall performance was computed using the Berkeley benchmark algorithm [1], which computes a score based on the F-measure. Table 2 shows the obtained score of our approach compared with other existing approaches (Note that like us, most of these approaches use training). One of the obtained soft boundary maps is shown in Figure 3.

Analyzing the top individuals might provide additional insights that could improve the selection of function and

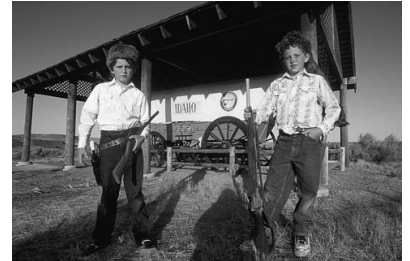

(a)

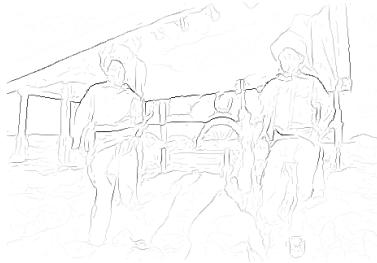

(b)
Figure 3: (a) Sample test image (b) Extracted soft boundary map, using our best evolved detector, with $F=0.81$

Table 2: Performance summary table of local boundary detectors

\begin{tabular}{lc}
\hline Method & Performance \\
\hline \hline Brightness and texture gradient & 0.63 \\
\hline GP/TG Detector & 0.62 \\
\hline OE/TG Detector & 0.61 \\
\hline $\begin{array}{l}\text { Learning of the brightness distribution } \\
\text { (brightness Gradient) }\end{array}$ & 0.60 \\
\hline GM/TG & 0.58 \\
\hline Texture gradient (TG) & 0.58 \\
\hline Multiscale gradient magnitude & 0.58 \\
\hline Second moment matrix & 0.57 \\
\hline Gradient magnitude (GM) & 0.56 \\
\hline $\begin{array}{l}\text { Segmentation induced by } \\
\text { scale invariance }\end{array}$ & 0.48 \\
\hline
\end{tabular}

terminal sets. Similarly, adding mid- and high-level cues that have been shown [2] to improve overall performance on the Berkeley benchmark [1] might also contribute to the evolutionary process. These, and the informed use of additional computational resources to improve performance, all constitute our short-term future work.

\section{REFERENCES}

[1] The berkeley segmentation dataset and benchmark www.cs.berkeley.edu/projects/vision/bsds/.

[2] P. Dollar, Z. Tu, and S. Belongie. Supervised learning of edges and object boundaries. IEEE Computer Society Conference on Computer Vision and Pattern Recognition, 2006.

[3] I. Kadar, O. Ben-Shahar, and M. Sipper. Evolving boundary detectors for natural images via genetic programming. In Proc. of the 19th International Conference on Pattern Recognition, Tampa, Florida, 2008.

[4] J. R. Koza, M. A. Keane, M. J. Streeter, W. Mydlowec, J. Yu, and G. Lanza. Genetic Programming IV: Routine Human-Competitive Machine Intelligence. Kluwer Academic Publishers, 2003.

[5] D. Martin, C. Fowlkes, and J. Malik. Learning to detect natural image boundaries using local brightness, color and texture cues. PAMI04, 2004.

[6] D. Matin, C. Fowlkes, D. Tal, and J. Malik. A database of human segmented natural images and its applications to evaluating segmentations algorithms and measuring ecological statistics. In Proc. of international Conference on Computer Vision, 2001. 